\title{
Channel Estimation Using Multi-stage Compressed Sensing for Millimeter Wave MIMO Systems
}

\author{
Baghdad Hadji ${ }^{1}$, Abdeldjalil Aïssa-El-Bey ${ }^{2}$ Lamya Fergani $^{1}$ and Mustapha Djeddou ${ }^{3}$ \\ ${ }^{1}$ LISIC Laboratory,University of Science and Technology Houari Boumediene, Algiers, Algeria \\ Email:hadjb.ebm@gmail.com, lamifer@msn.com \\ ${ }^{2}$ IMT Atlantique, UMR CNRS 6285 Lab-STICC, Brest F-29238, France \\ Email:abdeldjalil.aissaelbey@imt-atlantique.fr \\ ${ }^{3}$ École Militaire Polytechnique,B.P 17, Bordj el Bahri (16111), Algeria \\ Email:djeddou.mustapha@gmail.com
}

\begin{abstract}
Millimeter-wave (mmWave) and multiple-input multiple-output (MIMO) combination technologies have attracted extensive attention from both academia and industry for meeting future communication challenges and requirements. As a viable option to deal with the trade-off between hardware complexity and system performance, hybrid analog/digital architectures are regarded as efficient mmWave MIMO transceivers. While acquiring channel state information (CSI) is a challenging task to design the optimal beamformers/combiners, especially in mmWave communications due to a lot of challenges. Fortunately, the sparse nature of the channel allows to leverage the compressed sensing (CS) tools and theories. However, the critical challenge to develop a CS-based formulation for estimating the mmWave channel is the codebook design (sensing matrices) and its pilot symbol numbers. In this paper, we proposed a multistage CS-based algorithm to estimate the channel explicitly using pilot and data symbols which enable increasing the number of measurements to enhance the estimation accuracy and maximize the spatial diversity by reducing the overlapping between training beams. Simulations confirmed that our proposed method has the best results compared to the existing methods based on codebook schemes.
\end{abstract}

Index Terms-Millimeter-wave channel estimation, Multi-stage compressed sensing, MmWave MIMO transceiver, Detection algorithms, Greedy algorithms

\section{INTRODUCTION}

To accommodate the huge wireless communications capacity, millimeter-wave (mmWave) frequency band $(30-300 \mathrm{GHz})$ is considered as a potential candidate to host very high data rate communications [1], by the fundamental solving of the spectrum congestion problem [2], thanks to the large bandwidth available at these frequencies [3]. However, mmWave signals suffer from severe propagation loss, penetration loss and rain fading compared to signals in lower frequencies [4]. Therefore, large directional beamforming powered by multiple-input multiple-output (MIMO) systems is highly recommended at the transceiver to achieve high-quality communication links. Fortunately, the short-wavelength allows more antenna elements to be integrated into mmWave devices. Actually, due to the hardware complexity and power consumption constraints, hybrid analog/digital architectures are regarded as efficient transceivers for mmWave MIMO communication systems [5]. In this architecture, the MIMO processing is splitted into the analog and digital domains where a small number of radio frequency ( $R F$ ) chains are tied to a large antenna array. The number of RF chains can be reduced to equal the number of transmitted/received data streams, which is, generally, much lower than the number of antenna elements [6]. Moreover, optimal beamformers/combiners design is based on the availability of channel state information (CSI) at the transceiver. On the other hand, acquiring CSI in the mmWave MIMO system is hard due to a lot of challenges such as the large antenna array, the very low received signal-to-noise ratio (SNR), and the sparse scattering nature of mmWave channels. According to the sparsity of the mmWave channel matrix, methods and developments based on compressed sensing (CS) tools are mainly leveraged to estimate the mmWave channel. In [7], based on adaptive CS-based tools and a multi-resolution hierarchical codebook design, a hierarchical search algorithm is proposed for jointly estimating the different parameters of the channel paths, namely the angle of departure (AoD), the angle of arrival (AoA), and each path's gain. Although the hierarchical search algorithm can avoid an exhaustive beam search, its performance tends to be limited by the training beam patterns [8]. Besides, an excessive channel feedback requirement makes the adaptive $\mathrm{CS}$ approach less attractive in practical mmWave systems despite its robustness to noise [9]. Unlike the hierarchical search algorithm, the authors in [8] exploited an explicit channel estimation technique based on CS formulation that allows directly using the orthogonal matching pursuit (OMP) algorithm. the critical challenge to develop a CS-based formulation for estimating the mmWave channel is the codebook design and its pilot symbol number. As known, sensing random matrices provide an adequate constant mutual coherence and satisfy the restricted isometry property (RIP) condition that guarantees the exact sparse signal recovery or estimation. Therefore, randomly permuting the columns of hybrid beamformer/combiner matrix is widely used to generate the sensing matrices in order to enhance the CS-based mmWave channel estimator performance. On the other hand, using i.i.d. random vectors as pilots are difficult because the RF beamformers, which are employed to increase the SNR, yield directional training beams that are correlated with each other [8], which leads to decrease the performance. Also, the orthogonality of the deterministic pilots is limited by 
their number. The work in [10] proposed a precoder column ordering algorithm to achieve a lower mutual coherence where the pilot symbol columns are chosen from the discrete Fourier transform (DFT) matrix. However, the pilot orthogonality is affected, because the pilot columns size equal to RF chain numbers in hybrid mmWave MIMO systems as mentioned above.

In this work, we propose a multi-stage CS-based algorithm to estimate the channel for the hybrid mmWave MIMO transceiver. Our proposed method performs the explicit channel estimation using pilot and data symbols which allow the limitation of random pilot number in order to maintain the orthogonality of the training beams throughout the estimation process. Hence, reducing the effect of the overlapping between training beams leads to maximize spatial diversity. In addition, using the data symbols improve also the spectral efficiency of mmWave MIMO systems. Meanwhile, using data symbols increases the number of measurements and consequently augment the estimation accuracy.

\section{SYSTEM MODEL}

We consider time division duplexing (TDD) system protocols and downlink data transmission techniques for the mmWave point-to-point hybrid MIMO system shown in Fig. 1. The transmitter (Tx) is equipped with $N_{\mathrm{tx}}$ transmit antennas and $N_{R F}^{\mathrm{tx}}$ RF chains to send $N_{s}$ streams of data symbols whereas, the receiver (Rx) has $N_{r x}$ antennas and $N_{R F}^{r x} \mathrm{RF}$ chains. The number of data streams is constrained to be bounded at the $\mathrm{Tx}$ and $\mathrm{Rx}$ by $N_{s} \leq N_{R F}^{\mathrm{tx}} \leq N_{\mathrm{tx}}$ and $N_{s} \leq N_{R F}^{r x} \leq N_{\text {rx }}$, respectively. To transmit the symbols at the time instance $n$ in a hybrid architecture, the Tx applies a hybrid precoder $\boldsymbol{F}_{n} \in \mathbb{C}^{N_{\mathrm{t} \times} \times N_{s}}$ which is composed of an $N_{R F}^{\mathrm{tx}} \times N_{s}$ baseband precoder $\boldsymbol{F}_{B B, n}$ followed by an $N_{\mathrm{tx}} \times N_{R F}^{\mathrm{tx}} \mathrm{RF}$ precoder $\boldsymbol{F}_{R F, n}$, such that $\boldsymbol{F}_{n}=\boldsymbol{F}_{R F, n} \boldsymbol{F}_{B B, n}$. If $\boldsymbol{F}_{R F, n}$ realized by analog phase shifters, its entries are constrained to satisfy $\left(\left[\boldsymbol{F}_{R F, n}\right]_{:, i}\left[\boldsymbol{F}_{R F, n}\right]_{:, i}^{H}\right)_{\ell, \ell}=N_{\mathrm{tx}}^{-1}$, where $(\cdot)_{\ell, \ell}$ denotes the $\ell^{\text {th }}$ diagonal element, i.e., all entries are of equal norm. At the Rx, the hybrid combiner $\boldsymbol{W}_{n} \in \mathbb{C}^{N_{\mathrm{tx}} \times N_{s}}$ composed of an $N_{R F}^{\mathrm{rx}} \times N_{s}$ baseband combiner $\boldsymbol{W}_{B B, n}$ and an $N_{\text {rx }} \times N_{R F}^{\text {rx }} \quad \mathrm{RF}$ combiner $\boldsymbol{W}_{R F, n}$ is used to measure and to detect the instantaneous transmitted signal through a narrowband block-fading channel. Therefore, the received signal vector at the same time instance $n$ is given by

$$
\boldsymbol{y}_{n}=\sqrt{\gamma} \boldsymbol{W}_{n}^{H} \boldsymbol{H} \boldsymbol{F}_{n} \boldsymbol{s}_{n}+\boldsymbol{W}_{n}^{H} \boldsymbol{\eta}_{n}
$$

where $\gamma$ denotes the average transmit power, and $\boldsymbol{s}_{n} \in$ $\mathbb{C}^{N_{s} \times 1}$ is the instantaneous transmitted signal vector. $\boldsymbol{\eta}_{n} \sim$ $\mathcal{C N}\left(0, \sigma_{n}^{2} \boldsymbol{I}\right)$ is the additive noise vector, and $\boldsymbol{H} \in \mathbb{C}^{N_{\mathrm{rx}} \times N_{\mathrm{tx}}}$ represents the channel matrix.

For the mmWave MIMO channel, we adopt a widely used Saleh-Valenzuela model with limited $L$ paths as in [8] [10] [11], where the downlink channel can be expressed as

$$
\boldsymbol{H}=\sqrt{\frac{N_{\mathrm{rx}} N_{\mathrm{tx}}}{L}} \sum_{i=1}^{L} \alpha_{i} \boldsymbol{a}_{\mathrm{rx}}\left(\theta_{i}\right) \boldsymbol{a}_{\mathrm{tx}}^{H}\left(\phi_{i}\right)
$$

where $\alpha_{i}$ is the complex gain of the $i^{t h}$ path and it can define the channel type (Rayleigh, Rician or Nakagami), whereas $\phi_{i}$ and $\theta_{i} \in[0,2 \pi]$ are the $i^{t h}$ path's azimuth angles of departure or arrival (AoDs/AoAs) of the $\mathrm{Tx}$ and $\mathrm{Rx}$, respectively. Assuming only the azimuth angles and uniform linear array (ULA) configuration, the transmit and receive array response vectors corresponding to $\mathrm{AoD} / \mathrm{AoA}$, respectively, are written as

$$
\begin{aligned}
& \boldsymbol{a}_{\mathrm{tx}}\left(\phi_{i}\right)=\frac{1}{\sqrt{N_{\mathrm{tx}}}}\left[1, e^{j \frac{2 \pi}{\lambda} d \sin \left(\phi_{i}\right)}, \ldots, e^{j\left(N_{\mathrm{tx}}-1\right) \frac{2 \pi}{\lambda} d \sin \left(\phi_{i}\right)}\right]^{T} \\
& \boldsymbol{a}_{\mathrm{rx}}\left(\theta_{i}\right)=\frac{1}{\sqrt{N_{\mathrm{rx}}}}\left[1, e^{j \frac{2 \pi}{\lambda} d \sin \left(\theta_{i}\right)}, \ldots, e^{j\left(N_{\mathrm{rx}}-1\right) \frac{2 \pi}{\lambda} d \sin \left(\theta_{i}\right)}\right]^{T}
\end{aligned}
$$

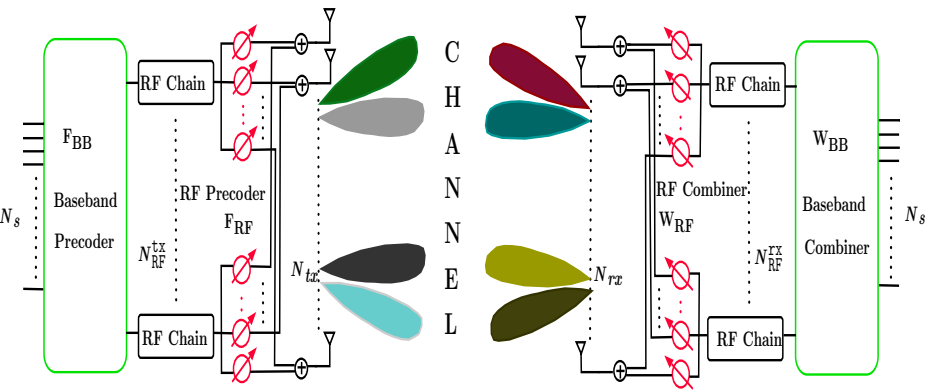

Fig. 1. hybrid beamforming architecture at the transceiver.

where $d$ denotes the distance between antenna elements, and $\lambda$ denotes the wavelength of the signal. The channel can be represented by matrix product form as

$$
\boldsymbol{H}=\boldsymbol{A}_{\mathrm{rx}} \operatorname{diag}(\boldsymbol{\alpha}) \boldsymbol{A}_{\mathrm{tx}}^{H}
$$

where $\boldsymbol{\alpha}=\left[\alpha_{1}, \ldots, \alpha_{L}\right]^{T}, \boldsymbol{A}_{\mathrm{tx}}=\left[\boldsymbol{a}_{\mathrm{tx}}\left(\phi_{1}\right), \ldots, \boldsymbol{a}_{\mathrm{tx}}\left(\phi_{L}\right)\right]$, and $\boldsymbol{A}_{\mathrm{rx}}=\left[\boldsymbol{a}_{\mathrm{rx}}\left(\theta_{1}\right), \ldots, \boldsymbol{a}_{\mathrm{rx}}\left(\theta_{L}\right)\right]$.

\section{Multi-Stage Compressed Sensing}

In this section, we present our method based on multi-stage CS approach to estimate the mmWave channel for the system shown in Fig. 1. To formulate the channel estimation problem, we assume that Tx sends $M$ known pilot followed by $N-M$ unknown data symbols. By vectorizing the right-hand side of the signal model in (1), the downlink received signal vector by Rx at the $n^{\text {th }}$ time is rewritten as follows:

$$
\begin{gathered}
\boldsymbol{y}_{n}=\sqrt{\gamma}\left(\boldsymbol{s}_{n}^{T} \boldsymbol{F}_{n}^{T} \otimes \boldsymbol{W}_{n}^{H}\right) \operatorname{vec}(\boldsymbol{H})+\overline{\boldsymbol{\eta}}_{n} \\
n=1, \ldots, N
\end{gathered}
$$

where $\overline{\boldsymbol{\eta}}_{n}$ is the noise vector after combining. To exploit the sparse nature of the channel, we adopt the concept of discrete virtual angular domain (VAD) channel model [12] to provide an approximation of $\boldsymbol{H}$ in (2) by assuming that the AoDs and AoAs are taken from grids that have size $G$, where $\phi_{i}, \theta_{i} \in$ $\left\{0, \frac{2 \pi}{G}, \ldots, \frac{2 \pi(G-1)}{G}\right\}$ with $G \gg L$. Then, the discrete virtual channel can be written as

$$
\boldsymbol{H}=\overline{\boldsymbol{A}}_{\mathrm{rx}} \overline{\boldsymbol{H}}_{\alpha} \overline{\boldsymbol{A}}_{\mathrm{t} \mathrm{x}}^{H}
$$

where $\overline{\boldsymbol{H}}_{\alpha} \in \mathbb{C}^{G \times G}$ is a channel matrix that has only $L$ nonzero elements in the positions corresponding to the AoAs and AoDs, $\overline{\boldsymbol{A}}_{\mathrm{rx}} \in \mathbb{C}^{N_{\mathrm{xx}} \times G}$ and $\overline{\boldsymbol{A}}_{\mathrm{tx}} \in \mathbb{C}^{N_{\mathrm{tx}} \times G}$ are dictionary 
matrices at the $\mathrm{Tx}$ and $\mathrm{Rx}$ respectively. Substituting (5) into (4) and exploit the properties of Kronecker product, i.e., $\operatorname{vec}(\boldsymbol{H})=\boldsymbol{A}_{D} \boldsymbol{h}_{\alpha}$, where $\boldsymbol{A}_{D} \in \mathbb{C}^{N_{\mathrm{rx}} N_{\mathrm{tx}} \times G^{2}}$ is dictionary matrix, such that $\boldsymbol{A}_{D}=\overline{\boldsymbol{A}}_{\mathrm{tx}}^{*} \otimes \overline{\boldsymbol{A}}_{\mathrm{rx}} . \boldsymbol{h}_{\alpha} \in \mathbb{C}^{G^{2} \times 1}$ is vector which contains the path gains of the channel matrix $\boldsymbol{H}$. We get

$$
\boldsymbol{y}_{n}=\sqrt{\gamma}\left(\boldsymbol{s}_{n}^{T} \boldsymbol{F}_{n}^{T} \otimes \boldsymbol{W}_{n}^{H}\right) \boldsymbol{A}_{D} \boldsymbol{h}_{\alpha}+\overline{\boldsymbol{\eta}}_{n}
$$

By stacking $N$ instantaneous received signal vector, we have

$$
\begin{aligned}
\tilde{\boldsymbol{y}}_{G} & =\sqrt{\gamma}\left[\boldsymbol{s}_{1}^{T} \boldsymbol{F}_{1}^{T} \otimes \boldsymbol{W}_{1}^{H}, \ldots, \boldsymbol{s}_{N}^{T} \boldsymbol{F}_{N}^{T} \otimes \boldsymbol{W}_{N}^{H}\right]^{T} \boldsymbol{A}_{D} \boldsymbol{h}_{\alpha}+\tilde{\boldsymbol{\eta}}_{G} \\
& =\sqrt{\gamma}\left[\boldsymbol{\psi}_{1}, \ldots, \boldsymbol{\psi}_{N}\right]^{T} \boldsymbol{A}_{D} \boldsymbol{h}_{\alpha}+\tilde{\boldsymbol{\eta}}_{G} \\
& =\sqrt{\gamma} \underbrace{\boldsymbol{\Psi} \boldsymbol{A}_{D}}_{\boldsymbol{\Phi}_{G}} \boldsymbol{h}_{\alpha}+\tilde{\boldsymbol{\eta}}_{G}
\end{aligned}
$$

where $\tilde{\boldsymbol{y}}_{G}=\left[\boldsymbol{y}_{1}^{T}, \ldots, \boldsymbol{y}_{N}^{T}\right]^{T}$ is the collected received signal. $\Psi_{G}=\left[\boldsymbol{\psi}_{1}, \ldots, \boldsymbol{\psi}_{N}\right]^{T}$ is the collected sensing matrix, where $\boldsymbol{\psi}_{n}=\boldsymbol{s}_{n}^{T} \boldsymbol{F}_{n}^{T} \otimes \boldsymbol{W}_{n}^{H}$, whereas $\boldsymbol{\Phi}_{G}$ is the total measurement matrix, and $\tilde{\boldsymbol{\eta}}_{G}=\left[\overline{\boldsymbol{\eta}}_{1}^{T}, \ldots, \overline{\boldsymbol{\eta}}_{N}^{T}\right]^{T}$ is the collected noise vector. Now, we divide the compressed sensing model in (8) to split the total measurement matrices into two measurement matrices, one correspond to the pilot and the other corresponds to unknown data symbols as given by

$$
\left[\begin{array}{c}
\boldsymbol{y}_{1} \\
\vdots \\
\boldsymbol{y}_{M} \\
\boldsymbol{y}_{M+1} \\
\vdots \\
\boldsymbol{y}_{N}
\end{array}\right]=\sqrt{\gamma}\left[\begin{array}{c}
\boldsymbol{\Phi}_{1} \\
\boldsymbol{\Phi}_{2}
\end{array}\right] \boldsymbol{h}_{\alpha}+\left[\begin{array}{c}
\overline{\boldsymbol{\eta}}_{1} \\
\vdots \\
\overline{\boldsymbol{\eta}}_{M} \\
\overline{\boldsymbol{\eta}}_{M+1} \\
\vdots \\
\overline{\boldsymbol{\eta}}_{N}
\end{array}\right]
$$

Therefore, we have two-stage, where the $M$ pilots are used in the first stage to estimate the channel where its received training signal model is given as

$$
\tilde{\boldsymbol{y}}_{1}=\boldsymbol{\Phi}_{1} \boldsymbol{h}_{\alpha}+\tilde{\boldsymbol{\eta}}_{1}
$$

In the second stage, we can exploit the estimated channel from the first stage to detect the unknown data, then the received signal model of the second stage is written as

$$
\tilde{\boldsymbol{y}}_{2}=\boldsymbol{\Phi}_{2} \boldsymbol{h}_{\alpha}+\tilde{\boldsymbol{\eta}}_{2}
$$

\section{A. First stage}

To estimate the channel at the first stage, we adopt the openloop channel estimator approach that performs explicit channel estimation. This approach applies the compressed sensing (CS) model that allows using the greedy algorithms to recover the sparse channel vector. Therefore, the estimated channel $\boldsymbol{h}_{\alpha}$ is obtained by solving the following problem:

$$
\begin{aligned}
& \hat{\boldsymbol{h}}_{\alpha}=\underset{\boldsymbol{h}_{\alpha}}{\arg \min }\left\|\tilde{\boldsymbol{y}}_{1}-\sqrt{\gamma} \boldsymbol{\Phi}_{1} \boldsymbol{h}_{\alpha}\right\|_{2} \\
& \text { subjet to }\left\|\boldsymbol{h}_{\alpha}\right\|_{0}=L
\end{aligned}
$$

The above optimization problem in (11) is a non-convex optimization with $\mathcal{L}_{0}$ norm and is difficult and intractable to solve. For this, we use the orthogonal matching pursuit (OMP) algorithm as a greedy CS algorithm to solve (11).

\section{B. Second stage}

After, we use the estimated channel in the second stage to detect the unknown data symbols. In our implementation, we used different detection techniques as least square (LS), Minimum mean square error (MMSE), minimum mean-squared error with successive interference cancellation (MMSE-SIC), simplicity [13], and semidefinite relaxation row-by-row (SDRRBR) [14]. According to the simulation results, we found that the SDR-RBR detector has better performance in the QPSK scenario. Due to this, we choose SDR-RBR to detect the unknown data symbols. As mentioned above, considering the $N-M$ unknown data symbols transmitted through the estimated channel, each received signal in 10 can be expressed as:

$$
\boldsymbol{y}_{d, n}=\sqrt{\gamma} \underbrace{\boldsymbol{W}_{d, n}^{H} \hat{\boldsymbol{H}} \boldsymbol{F}_{d, n}}_{\boldsymbol{\Theta}} \boldsymbol{s}_{d, n}+\overline{\boldsymbol{\eta}}_{d, n}
$$

where $\boldsymbol{F}_{d, n}, \boldsymbol{W}_{d, n}$ are the precoder and combiner that are used in second stage to send the unknown data, $\boldsymbol{s}_{d, n} \in \mathbb{C}^{N_{R F}^{\mathrm{tx}} \times 1}$ is the unknown data vector, and $\overline{\boldsymbol{\eta}}_{d, n}$ is noise vector after combining at Rx. The MIMO data detection problem in the symbol constellation set $\mathcal{S}=\{ \pm 1 \pm j\}$ is equivalent to:

$$
\hat{\boldsymbol{s}}_{d, n}=\underset{\boldsymbol{s}_{d, n} \in \mathcal{S}}{\arg \min }\left\|\boldsymbol{y}_{d, n}-\sqrt{\gamma} \boldsymbol{\Theta} \boldsymbol{s}_{d, n}\right\|_{2}
$$

The problem in 13 can be solved by maximum likelihood (ML) detection for yielding the minimum error probability in the data detection process during the second stage. However, ML detection has high computational complexity due to the exhaustively searching for all the candidate vectors. Therefore, SDR-RBR detector deals with the ML detection problem, especially with BPSK/QPSK constellation to reduce the complexity. To apply the SDR-RBR solution, we convert the model in (12) to an equivalent real-valued system as follow:

$$
\boldsymbol{y}_{c, n}=\sqrt{\gamma} \boldsymbol{\Theta}_{c} \boldsymbol{s}_{c, n}+\overline{\boldsymbol{\eta}}_{c, n}
$$

where $\boldsymbol{y}_{c, n} \in \mathbb{R}^{2 N_{R F}^{\mathrm{r} x} \times 1}$ is the real-valued received signal vector, $\boldsymbol{\Theta}_{c} \in \mathbb{R}^{2 N_{R F}^{\mathrm{rx}} \times 2 N_{R F}^{\mathrm{tx}}}$ is the real-valued matrix version of $\boldsymbol{\Theta}$, whereas $\boldsymbol{s}_{c, n} \in \mathbb{R}^{2 N_{R F}^{\mathrm{tx}} \times 1}$ is real-valued unknown data vector, and $\boldsymbol{n}_{c, n} \in \mathbb{R}^{2 N_{R F}^{r \times} \times 1}$ is real-valued additive Gaussian noise vector. Then, the SDR problem can be written as

$$
\begin{gathered}
\hat{\boldsymbol{s}}_{c, n}=\underset{\boldsymbol{s}_{c, n} \in \mathcal{S}^{2 N_{R F}^{r x}}}{\arg \min }\left\{\operatorname{Tr}\left(\boldsymbol{\Theta}_{c}^{T} \boldsymbol{\Theta}_{c} \boldsymbol{S}\right)-2 \boldsymbol{s}_{c, n}^{T} \boldsymbol{\Theta}_{c}^{T} \boldsymbol{y}_{c, n}+\left\|\boldsymbol{y}_{c, n}\right\|_{2}^{2}\right\} \\
\text { subject to } \quad \boldsymbol{S} \succeq \boldsymbol{s}_{c, n}^{T} \boldsymbol{s}_{c, n} \\
S_{i i}=1, i=1, \ldots, 2 N_{R F}^{r x}
\end{gathered}
$$

We adopt the row-by-row (RBR) method [14] to solve this SDR problem. 


\section{Last stage}

Finally, we exploit the pilot of the first stage and the detected data in the second stage into the CS model in (8) to refine the re-estimation of the mmWave channel by solving:

$$
\begin{gathered}
\tilde{\boldsymbol{h}}_{\alpha}=\underset{\substack{\boldsymbol{h}_{\alpha} \\
\text { subjet to }}}{\arg \min }\left\|\tilde{\boldsymbol{h}}_{\alpha}\right\|_{0} \leq L \\
\end{gathered}
$$

In the last stage, it appears that we have more measurement numbers which leading to perform the recovery process of the true support of $\boldsymbol{h}_{\alpha}$. As the augmented of the row numbers of the noise vector, we exploit the gOMP algorithm to finish the mmWave channel re-estimation with fast processing speed and competitive computational complexity [15]. Algorithm [1 summarizes all algorithms that are used in each stage.

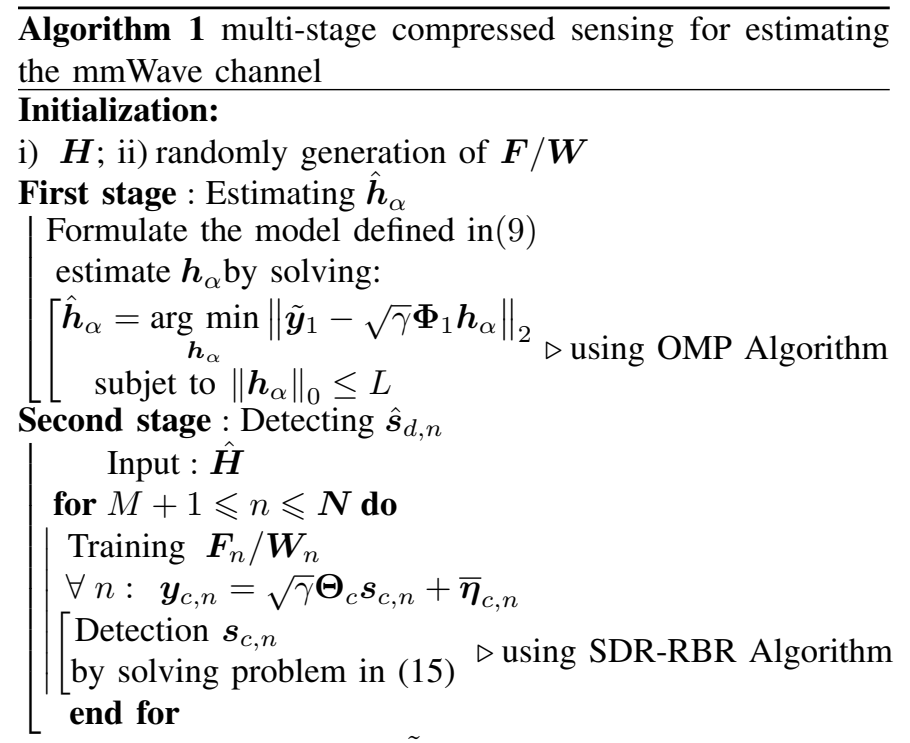

Last stage : Re-estimating $\tilde{\boldsymbol{h}}_{\alpha}$

Formulate the model defined in (7)

re-estimate $\boldsymbol{h}_{\alpha}$ by solving:

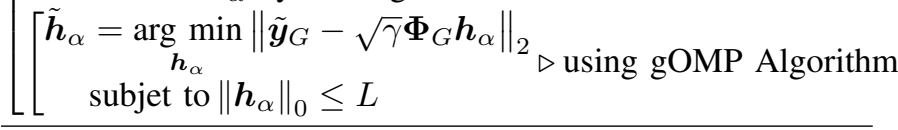

\section{SIMULATION RESULTS}

In this section, we provide numerical simulation results to evaluate each stage performance of our proposed channel estimator algorithm for the hybrid MIMO system. We assume that the transceiver is equipped with $N_{\text {tx }}=N_{\text {rx }}=32$ and $N_{R F}^{\mathrm{tx}}=N_{R F}^{\mathrm{rx}}=2$. The downlink channel has $L=9$ paths and Rayleigh distributed, where the azimuth AoAs and AoDs are uniformly distributed in $[0,2 \pi]$, and the noise power is $\sigma=-173+10 \log _{10}\left(\boldsymbol{B}_{w}\right)$ with transmission bandwidth $\boldsymbol{B}_{w}=100 \mathrm{MHz}$. The hybrid training precoders/combiners are randomly generated using 6 quantization bits to design of $\mathrm{RF}$ phases shifters. At the $\mathrm{Tx}$ and $\mathrm{Rx}$, the number of angle grids is assumed $G=160$. We used the normalized mean squared error (NMSE) defined as $\mathbb{E}\left[\|\boldsymbol{H}-\hat{\boldsymbol{H}}\|_{F}^{2} /\|\boldsymbol{H}\|_{F}^{2}\right]$, where $\boldsymbol{H}$ and $\hat{\boldsymbol{H}}$ are the true channel and the estimated channel, respectively. The number of pilots used at first stage equal 100. In addition, we provide the symbol error rate (SER) performance comparison to evaluate the detection of the unknown data transmitted by the QPSK modulation scheme.

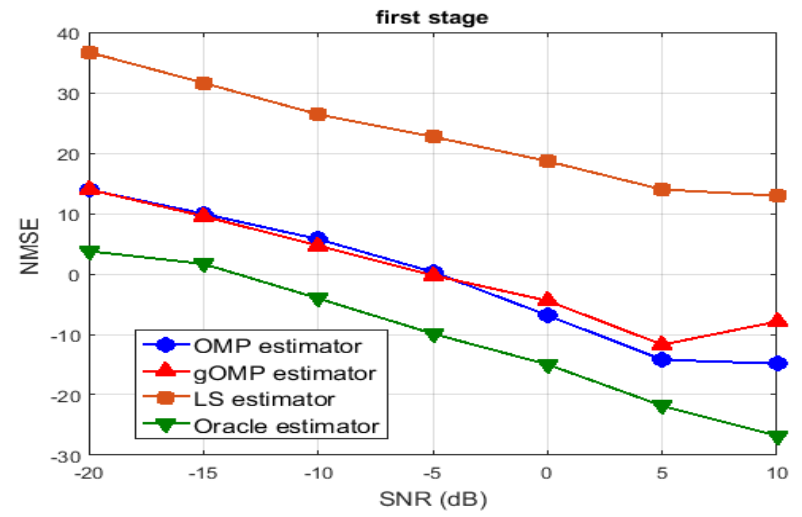

Fig. 2. NMSE performance of estimated at the first stage v.s. SNR for the LS, OMP, gOMP, and Oracle estimators

Fig. 2 shows the NMSE performance of the channel estimated by the greedy algorithms (OMP, gOMP) versus SNR and compared with the LS and oracle estimators results. The LS method has the worst results because of the underdetermination of the model system in (9) that raised from the fewer measurement numbers than the product of antenna in the formulation of the total sensing matrix. The oracle estimator has the best NMSEs results which make it a lower bound in the comparison of other methods results, due to the prior knowledge of AoDs/AoAs. Whereas, the greedy algorithms show good outcomes with a similar trend to the oracle estimator because of their capability to enhance the recovery sparsity, but, from $5 \mathrm{~dB}$ SNR, their results remain almost constant due to the small measurements, i.e., the limited pilot numbers.

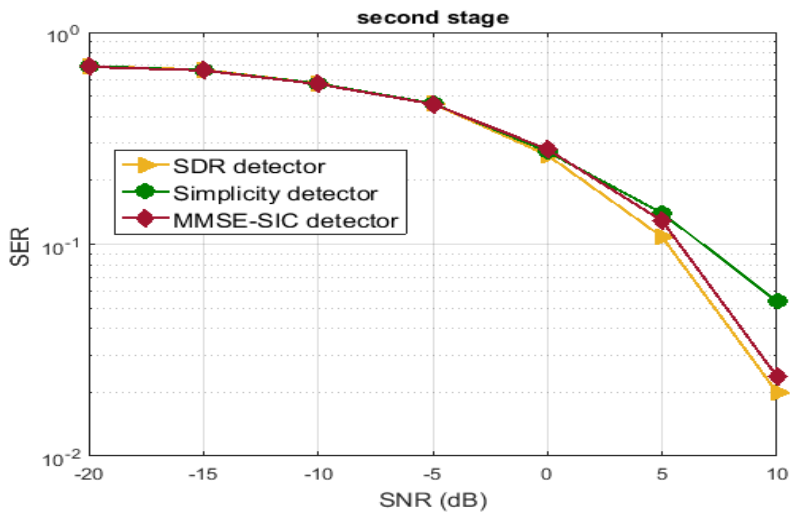

Fig. 3. Comparison of the SER performance of the different detectors used at the second stage

As mentioned above, the estimated channel in the first stage is used to transmit the unknown data. Fig. 3 compares SER of SDR-RBR, MMSE-SIC, and Simplicity detectors versus SNR. Notably, the results show that the SDR-RBR is better and outperforms others as expected, especially, in QPSK scenario. 
As known, a detector that has a good SER performance require usually highly computational complexity. Therefore, the SDRRBR algorithm is enough to detect data in our case with low complexity. After, the detected data symbols are re-used as beam training to re-estimate the channel at the last stage.

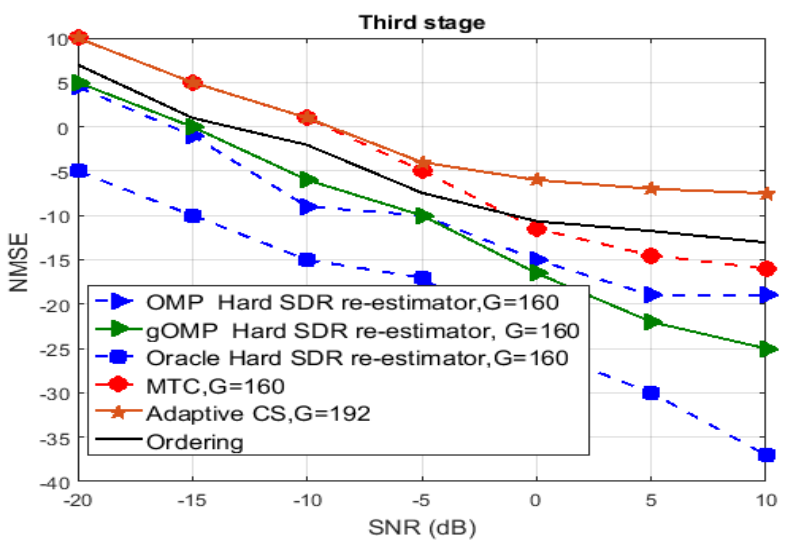

Fig. 4. Spectral efficiency v.s. SNR, achieved by different precoding methods.

Using detected data offers more measurements to improve the estimation of the mmWave channel without any correlation between the training beams. Fig. 4 illustrates the NMSE performance comparison of channel estimation for the last stage of our multi-stage CS-based algorithm, codebook based ordering scheme [10], codebook based minimal total coherence scheme (MTC) [8], hierarchical codebook based adaptive CS scheme [7], and oracle scheme. From this figure, we can see that the NMSE performance of our proposed scheme outperforms the other codebook schemes although the use of the same quantized angle grids $(G=160)$. The oracle estimator still maintains good results as the lower bound. However, the adaptive CS scheme has lower results with grid size $G=192$ due to it needs a large number of the grid size. Even with more grid size, the angle estimation error is probably larger than the angle resolution (the maximum estimation error) in theory even if there are no hardware constraints and SNR is large enough [16]. Also, the last stage outperformed channel estimation results with $10 \mathrm{~dB}$ compared to the first stage thanks to the using of detected data which increase the measurements.

\section{CONCLUSION}

In this paper, we proposed a multi-stage CS-based algorithm to estimate the channel explicitly for the hybrid mmWave MIMO system. Our proposed method allows the exploitation of the detected data symbols as training beams to increases the number of measurement sensing matrix in order to improve the performance of the greedy algorithms that are used to estimate the channel. Moreover, our proposed method used simple algorithms in its different stages. Numerical simulations confirmed that our proposed method has the best results compared to the existing methods based on codebook schemes using the same quantized angle grids. The total sensing matrix design and performance synthesis will be considered in our future work.

\section{REFERENCES}

[1] M. G. Sanchez, "Millimeter-wave (mmwave) communications," Electronics, vol. 9, no. 2:251, 2020. https://doi.org/10.3390/ electronics9020251

[2] Z. Wang, M. Li, Q. Liu, and A. L. Swindlehurst, "Hybrid precoder and combiner design with low-resolution phase shifters in mmWave MIMO systems," IEEE Journal of Selected Topics in Signal Processing, vol. 12, no. 2 , pp. 256-269, 2018

[3] S. Noh, M. D. Zoltowski, and D. J. Love, "Multi-resolution codebook based beamforming sequence design in millimeter-wave systems," in 2015 IEEE Global Communications Conference (GLOBECOM), pp. 16, 2015.

[4] C. G. Tsinos, S. Chatzinotas, and B. Ottersten, "Hybrid analog-digital transceiver designs for multi-user MIMO mmWave cognitive radio systems," IEEE Transactions on Cognitive Communications and Networking, vol. 6, no. 1, pp. 310-324, 2019.

[5] F. Zhu, S. He, R. Li, Y. Huang, and X. You, "Energy-efficient hybrid precoding for broadband millimeter wave communication systems," in 2017 9th International Conference on Wireless Communications and Signal Processing (WCSP), pp. 1-5, 2017.

[6] K. Ardah, G. Fodor, Y. C. Silva, W. C. Freitas, and A. L. de Almeida, "Hybrid analog-digital beamforming design for SE and EE maximization in massive MIMO networks," IEEE Transactions on Vehicular Technology, vol. 69, no. 1, pp. 377-389, 2019.

[7] A. Alkhateeb, O. El Ayach, G. Leus, and R. W. Heath, "Channel estimation and hybrid precoding for millimeter wave cellular systems," IEEE Journal of Selected Topics in Signal Processing, vol. 8, no. 5 , pp. 831-846, 2014.

[8] J. Lee, G.-T. Gil, and Y. H. Lee, "Channel estimation via orthogonal matching pursuit for hybrid MIMO systems in millimeter wave communications," IEEE Transactions on Communications, vol. 64, no. 6, pp. 2370-2386, 2016.

[9] Y. Han and J. Lee, "Two-stage compressed sensing for millimeter wave channel estimation," in 2016 IEEE International Symposium on Information Theory (ISIT), pp. 860-864, 2016.

[10] J. Sung and B. L. Evans, "Hybrid beamformer codebook design and ordering for compressive mmWave channel estimation," in 2020 International Conference on Computing, Networking and Communications (ICNC), pp. 914-919, 2020.

[11] L. Liang, W. Xu, and X. Dong, "Low-complexity hybrid precoding in massive multiuser MIMO systems," IEEE Wireless Communications Letters, vol. 3, no. 6, pp. 653-656, 2014.

[12] C.-R. Tsai, Y.-H. Liu, and A.-Y. Wu, "Efficient compressive channel estimation for millimeter-wave large-scale antenna systems," IEEE Transactions on Signal Processing, vol. 66, no. 9, pp. 2414-2428, 2018.

[13] Z. Hajji, K. Amis, and A. Aïssa El Bey, "Iterative receivers for largescale MIMO systems with finite-alphabet simplicity-based detection," IEEE Access, vol. 8, pp. 21742-21758, 2020.

[14] H.-T. Wai, W.-K. Ma, and A. M.-C. So, "Cheap semidefinite relaxation MIMO detection using row-by-row block coordinate descent," in 2011 IEEE International Conference on Acoustics, Speech and Signal Processing (ICASSP), pp. 3256-3259, 2011.

[15] J. Wang, S. Kwon, and B. Shim, "Generalized orthogonal matching pursuit," IEEE Transactions on signal processing, vol. 60, no. 12, pp. 6202-6216, 2012.

[16] J. Yang, Z. Wei, N. Li, L. Sang, and P. Li, "Enhanced multi-resolution hierarchical codebook design for adaptive compressed sensing based millimeter wave channel estimation," in 2016 IEEE/CIC International Conference on Communications in China (ICCC), pp. 1-5, 2016. 